\title{
SEASONAL VARIATION OF SATURN'S AURORAL RADIO EMISSIONS IN 2004-2015: THE CORRELATION WITH SOLAR WIND ACTIVITY AND SOLAR EUV FLUX
}

\author{
A. Sasaki*, Y. Kasaba*, T. Kimura ${ }^{\dagger}$, C. Tao ${ }^{\ddagger}$, L. Lamy $^{\S}$, and B. Cecconi ${ }^{\S}$
}

\begin{abstract}
Saturn emits intense radio emissions, Saturn Kilometric Radiation (SKR), from the northern and southern polar regions at $3-1200 \mathrm{kHz}$. SKR is generated by fieldaligned energetic auroral electrons via the Cyclotron Maser Instability (CMI) at the local cyclotron frequency. Evaluation of Saturn's rotation period is based on the occurrence period of SKR because the SKR source is fixed in the planetary magnetic field with highly anisotropic beaming and forms a corotating searchlight of radio emission. For Saturn's magnetic field direction, the right-handed circularly polarized $(\mathrm{RH})$ emissions are from the northern region and the left-handed ( $\mathrm{LH})$ ones from the southern region. Cassini observations in the southern summer (2004-2009) showed that the period of SKR daily variation is variable [Kurth et al., 2008, JGR 113, A05222]. It was slightly longer in the southern (summer) hemisphere [Gurnett et al., 2009, Geophys. Res. Lett. 36, L16102], but close to each other near the equinox (September 2009) [Gurnett et al., 2010, Geophys. Res. Lett. 37, L24101]. We also studied the flux variation between northern and southern SKR in 2004-2010, and show that the LH (summer, south) is stronger than the RH (winter, north) on average [Kimura et al., 2013, JGR 118, 7019-7035]. Those characteristics could be related to the north-south asymmetry in the polar ionospheric conductivities, which are related to the seasonal variations of the solar EUV flux illuminating the polar region. However, its comprehensive explanation has not yet been established. In 2010-2013, the observations during the northern summer also show northern and southern SKR periods merge together without clear separation [Provan et al., 2014, JGR 119, 7380-7401; Fischer et al., 2015, Icarus 254, 72-91].

In this study, we extend our last SKR flux variation study from 2004-2010 [Kimura et al., 2013, JGR 118, 7019-7035] toward the northern summer (-2015, DOY 264). We note that the simple extension of the analysis period is not adequate because of the bias in the Cassini orbit. Since the SKR is stronger on the dawn side, we only used the data when Cassini was at the dawn side ( 2 h-10 h LT). And, in
\end{abstract}

\footnotetext{
* Department of Geophysics, Tohoku University, Sendai, Japan

$\dagger$ RIKEN Nishina Center for Accelerator-Based Science, Tokyo, Japan

$\ddagger$ National Institute of Information and Communications Technology (NICT), Japan

$\S$ LESIA, Observatoire de Paris, Meudon, France
} 
order to avoid the visibility effect of SKR caused by its propagation, we also limited the data by Cassini's latitude $(-5$ to $+30 \mathrm{deg}$. $(\mathrm{RH}),+5$ to $-30 \mathrm{deg}$. $(\mathrm{LH}))$ and the distance from Saturn $\left(10-100 \mathrm{R}_{S}\right)$. However, because Cassini's apokrone after 2007 was gradually shifted from dawn to dusk, the same criteria prevents from collecting enough dataset for the analysis.

For this study, we kept the same latitude and distance criteria but didn't adopt a LT condition. In the data when Cassini was close from the equator, both northern and southern SKR are observed simultaneously. Therefore we selected the data when Cassini was in the latitude within $\pm 5^{\circ}$ and verified the result. The variation of SKR peak intensity was evaluated by a running median with a window of \pm 35 days. In this result, the intensity of LH component in 2004-2009 (south, summer) was $\sim 10 \mathrm{~dB}$ stronger than $\mathrm{RH}$ (north, winter), which is consistent with the result in Kimura et al. [2013, JGR 118, 7019-7035]. In 2010-2012 both SKR intensities got close to each other. After 2013, RH (north, summer) was stronger by a few $\mathrm{dB}$ than LH (south, winter). Those variations of the flux ratio between northern and southern SKR after 2010 seem to be linked with those of the northern and southern SKR periods. We also note that the flux ratio was more than 10 in southern summer but only $2.5-5$ in northern summer, in the analyzed term.

In this paper, we will also show the correlations of the SKR flux variations to the solar activity, solar EUV flux in 2004-2015, as extension of the results from 2004-2010 done by Kimura et al. [2013, JGR 118, 7019-7035]. 